Nouvelles perspectives en sciences sociales

Revue internationale de systémique complexe et d'études relationnelles

Mediatization. Concepts, Changes, Consequences, Knut Lundby (dir.), New York, Peter Lang Pulblishing, 2009. 317 p.

\title{
Osée Kamga
}

Volume 7, numéro 2, mai 2012

URI : https://id.erudit.org/iderudit/1013062ar

DOI : https://doi.org/10.7202/1013062ar

Aller au sommaire du numéro

Éditeur(s)

Prise de parole

ISSN

1712-8307 (imprimé)

1918-7475 (numérique)

Découvrir la revue

Citer ce compte rendu

Kamga, O. (2012). Compte rendu de [Mediatization. Concepts, Changes,

Consequences, Knut Lundby (dir.), New York, Peter Lang Publishing, 2009. 317

p.] Nouvelles perspectives en sciences sociales, 7(2), 297-306.

https://doi.org/10.7202/1013062ar d'utilisation que vous pouvez consulter en ligne. 


\section{Compte rendu de lecture}

\section{Mediatization. Concepts, Changes, Consequences}

Knut Lundby (dir.), New York, Peter Lang Publishing, 2009. 317 p.

Par Osée Kamga Université de Sudbury

i les médias ont été étudiés en long, en large et en profondeur $\checkmark$ dans les dernières décennies, il n'y a cependant pas eu un concept fédérateur autour duquel structurer l'ensemble des travaux. Tel est l'a priori sur lequel repose le collectif Mediatization. Concept, Changes, Consequences (2009) dirigé par Knut Lundby. L'ouvrage est divisé en trois sections. La première, intitulée "Concept » se penche sur le concept de médiatisation, son émergence, ses contours et même sa portée; la seconde partie, "Changes", examine les changements majeurs sur les plans social et culturel, là où les processus de médiatisation sont à l'œuvre. La troisième partie, "Consequences", est consacrée à l'impact de la médiatisation dans des champs spécifiques de l'activité sociale. Ce compte rendu en présente les principales articulations. 


\section{Un monde médiatisé}

Dès son chapitre introductif, Knut Lundby dresse le constat selon lequel les médias de communication constituent un rouage essentiel du fonctionnement de la société contemporaine. Les médias transforment le visage même des diverses institutions sociales, qu'il s'agisse de la politique du sport ou de la religion, et modèlent la vie des individus qui, par ailleurs, doivent inclure une variété de médias dans leurs pratiques quotidiennes (p. 2). Constat qui revient d'ailleurs chez nombre de contributeurs, et notamment chez Krotz qui estime qu'il est désormais impossible d'analyser quelque institution sociale que ce soit en dehors de la dimension médiatique (p. 22). Chez Thomas, même l'individualisme moderne procède de la médiatisation, tant nombreux sont les discours, technologies et les pratiques qui l'évoquent, le produisent et le reproduisent (p. 271). Sur ce plan, Stig Hjarvard est plus précis quand il propose le concept "d'individualisme mou ". Dans son chapitre "Soft Individualism: Media And The Changing Social Character ", il montre comment, en raison de la prolifération des médias de masse et des médias interactifs, les individus vivent de plus en plus en contact immédiat avec la société. Comme résultat, le "métabolisme » entre la formation des habitudes individuelles et celles collectives augmente (p. 70). Ainsi, l'individualisme ambiant est fondamentalement tributaire du monde extérieur (p. 175). Stewart Hoover dans son chapitre "Complexities: The Case of Religious Cultures " prend appui notamment sur la montée du néo-évangélisme américain avec ses charismatiques télévangélistes, ainsi que sur la révolution iranienne, pour conclure que la religion de la modernité avancée est un phénomène médiatique.

L'inéluctabilité des médias dans l'activité et les processus sociaux explique la position que prennent Norm Friesen et Theo Hug dans leur chapitre "The Mediatic Turn: Exploring Concepts for Media Pedagogy "Ils renvoient dos à dos l'éducation aux médias comme moyen de se prémunir contre l'influence néfaste des médias et le "media litteracy" soit la maîtrise des technologies médiatiques dans la formation universitaire. Au-delà de leur 
présence ubiquitaire, les médias représentent l'a priori ou précondition épistémologique aussi bien du savoir quotidien que du savoir spécialisé (p. 80). Norm Friesen et Theo Hug estiment que la conscience de cet a priori doit être centrale dans la démarche pédagogique. Maren Hartman reprend le concept de « domestication " développé notamment par Roger Sylverstone, concept qui renvoie aux processus grâce auxquels les familles adoptent les médias, et soutient que c'est en particulier dans la vie quotidienne qu'on peut observer le processus de médiatisation à l'œuvre. Pour elle, la médiatisation et la domestication étant deux processus hautement intégrés, l'un se saurait être analysé sans l'autre (p. 238).

\section{La médiatisation : définition et pertinence}

Dans le premier chapitre de l'ouvrage, "Mediatization: A Concept with Which to Grasp Media and Society ", Friedrich Krotz propose une définition du concept de médiatisation. Définition qu'endossent nombre de contributeurs. La médiatisation est pour Krotz un méta-processus aussi fondamental que le processus d'individualisation ou de commercialisation et, par conséquent, elle ne saurait s'appréhender que dans la durée, avec des phases historiques et des réalisations spécifiques propres à chaque société (p. 24). Ainsi la médiatisation peut se définir comme :

a meta-process that is grounded in the modification of communication as the basic practice of how people construct the social and cultural world. They do so by changing communication practices that use media and refer to media. Hence, mediatisation is not a technologically driven concept, since it is not the media as technology that are causal, but the changes in how people communicate when constructing their inner and exterior realities by referring to media (p. 25, souligné dans le texte).

On aura noté que le moteur du processus en question, ce sont les manières de communiquer plutôt que les technologies de communication. Pour Krotz, le concept de médiatisation est d'autant plus pertinent qu'il rend mieux compte de la complexité et de la portée du rôle des médias dans le processus de socialisation que ceux de "mediation" ou de "medialization" par exemple. Non seulement il est plus facile à comprendre, estime 
Krotz, mais en plus on peut le mettre en dialogue avec des théories sociales comme celle de l'action communicationnelle de Habermas; avec des concepts comme ceux de " capital culturel » ou de "violence symbolique " chez Bourdieu, ou même celui de "figuration " chez Elias qui pose l'interdépendance à la base de l'expérience et de l'action humaine (p. 34-36). La pertinence du concept est aussi soulignée par Synne Skjulstad dans son chapitre "Dressing Up: The Mediatization of Fashion Online " qui analyse l'industrie de la mode dans son déploiement en ligne et son renouvellement perpétuel sous l'angle de transformations culturelles. Pour elle, "the concept of mediatization captures how fashion is spread and represented online and now articulated as multimodal online discourse" (p. 180). Krotz voit en la médiatisation un cadre conceptuel utile pour mener la recherche empirique. Ainsi par exemple peut-on analyser au niveau micro l'impact de la médiatisation sur l'individu, au niveau méso, son impact sur certaines institutions ou même sur les partis politiques, et au niveau macro, sur tout un champ d'activités comme la communication politique et la démocratie, la communication économique, les relations sociales ou même le champ de l'amusement (p. 31, 32). C'est peut-être dans cette efficacité opératoire que réside la faiblesse du concept, c'est-à-dire sa versatilité. Car, s'il ne renvoie en définitive à rien de spécifique, il y a risque de galvaudage, c'est-à-dire de perte de substance qui, à terme, le rendrait peu utile.

Reste que la pertinence ou même son utilité ne fait pas l'unanimité chez les chercheurs. Comme le rappelle Rothenbuhler dans son chapitre "Continuities: Communicative Form and Institutionalization ", Couldry en critique l'usage dans la théorisation du changement social, parce que, pour lui, il comporte l'idée d'un processus linéaire, unidirectionnel, parce qu'il semble suggérer qu’initialement il y a eu des processus sociaux naturels que les médias ont par la suite changés (p. 279). 


\section{Perspectives multiples}

Si la plupart des contributeurs de ce volume évitent une posture déterministe, il en va autrement d'Andrea Schrott. Dans son chapitre intitulé "Dimensions: Catch-All Label or Technical Term ", elle s'intéresse au mécanisme même de la médiatisation. Elle veut comprendre comment et dans quelles circonstances les médias modernes affectent ou même changent les structures sociales (p. 42). Schrott définit la médiatisation comme « a social process of media induced social change that functions by specific mechanism. This mechanism is the institutionalization of media logic in social spheres that were previously considered to be separated from the mass media " (p. 47). Pour elle, la recherche empirique devrait tenter d'évaluer le degré de changement induit par les médias et identifier les mécanismes grâce auxquels s'est institutionnalisée la logique médiatique. On commence ainsi à comprendre le sens de son intertitre "An Alternative Definition of Mediatization" (p. 46). D'une part, contrairement à Krotz et quelques auteurs du collectif, Schrott ne craint pas de verser dans une vision déterminisme en affirmant que les changements sociaux sont induits par les médias. D'autre part, elle postule une logique médiatique, une sorte de principe régulateur, qui opère dans le processus de médiatisation. Or, une telle perspective est remise en cause par plus d'un contributeur. Knut Lundby par exemple, dans son chapitre "Media Logic: Looking for Social Interaction ", estime que le concept de "logique médiatique ", par trop générique, occulte la variété des transformations inhérentes au processus de médiatisation et, par le fait-même, affaiblit l'argumentation centrale de la médiatisation. Le chapitre d'Andreas Hepp, "Differentiation: Mediatization and Cultural Change ", abonde dans le même sens et soutient qu'il est erroné de supposer une logique médiatique unique. Pour ce dernier, il faudrait plutôt étudier les interrelations concrètes entre la médiatisation et les changements culturels selon les contextes et les champs spécifiques (p. 154). Pour Eric Rothenbuhler, s'il faut envisager la médiatisation en termes d'une continuité théorique qui reconnaît que l'activité sociale s'est historiquement organisée 
autour des systèmes de communication, il faut tout aussi bien l'envisager en termes de processus hétérogène qui reconnaît la spécificité historique, sociale et même institutionnelle de chaque circonstance (p. 277). Un point de vue partagé par Krotz qui affirme :

The "media logic " of TV today is not the same as of a decade ago, and the "media logic" of a mobile phone is quite different for a 14-year-old girl as compared to a 55-year-old banker. Thus, there is no media logic independent of cultural and societal contexts and independent of history (p. 26).

Aussi, la posture qu'adopte André Jansson dans son chapitre "Mobile Belongings: Texturation and Stratification in Mediatization Processes » est fort intéressante dans la mesure où elle se démarque quelque peu de nombre de contributions. Jansson tente de saisir ce que représentent les médias interactifs pour les individus en situation migrante. Il montre alors que les communications médiatisées, non seulement ne remplacent que de manière limitée les contacts directs, mais quelquefois accroissent le besoin de ces contacts (p. 258). Plus généralement, il rappelle que le processus de médiatisation suit dans une large mesure les stratifications sociales existantes où le contrôle du flux communicationnel est dominé par ceux qui ont déjà le contrôle du capital culturel et économique (p. 249, 250). De ce point de vue, la médiatisation apparaît moins fondamentale comme facteur de changement que chez Krotz par exemple. Il faut rappeler que ce dernier, s'appuyant notamment sur les logiciels de correction de texte, estimait que même le capital culturel (dans le cas d'espèce le fait de savoir écrire correctement) n'était pas à l'abri du pouvoir transformateur de la médiatisation (p. 34).

On voit dès lors que les perspectives développées dans cet ouvrage ne sont pas uniformes, que les points de vue ne sont pas unanimes, ni sur la définition du concept, ni sur les contours de son objet. Knut Lundby le reconnaît dans sa conclusion quand il affirme: "This collection takes an inclusive approach to mediatization without homogenizing " (p. 293). Les perspectives se croisent, se répondent et quelquefois se contredisent, une diversité de pistes et de réflexions qui ajoute à l'intérêt de ce volume 
dont bénéficieront grandement étudiants et jeunes chercheurs en médias et société.

\section{La question du déterminisme}

Une théorie des médias ne saurait faire l'économie d'une réflexion sur la question du déterminisme. Elle est par conséquent omniprésente dans l'ouvrage et traverse les postures épistémologiques. Les uns, à l'instar de Krotz ou Lundby, fonctionnent avec l'a priori selon lequel le moteur du changement, ce sont les manières de communiquer plutôt que les appareils et dispositifs médiatiques. Les autres placent les médias de communication au centre du processus. Norm Friesen et Theo Hug par exemple, à la suite de Knuth Hicketier, postulent le caractère fondamentalement transformateur des médias. Pour eux, ces dispositifs structurent notre conscience du temps, notre attention et nos émotions et fournissent les moyens d'exprimer nos pensées; ils constituent à la fois le terrain du savoir et le savoir lui-même (p. 66). Strömbäck et Frank Esser, dans leur chapitre "Shaping Politics: Mediatization and Media Interventionism ", analysent comment les médias bouleversent le politique. Certes, ils affirment qu'ils entendent par média tout le système social et culturel de production, de diffusion, de circulation et de dissémination des symboles, signes, messages et valeurs (p. 209). Cependant, les exemples qu'ils prennent pour illustrer le concept d'interventionnisme médiatique (media interventionism) qu'ils présentent comme central pour comprendre la médiatisation de la politique, renvoient essentiellement à l'appareil médiatique et à ses acteurs (p. 217). La question du déterminisme est présente chez Rothenbuhler, en particulier dans son analyse du concept de logique médiatique. Rothenbuhler suggère que s'il existait une logique médiatique, les mêmes médias, dans des contextes différents, produiraient le même résultat. S'appuyant sur des exemples historiques et actuels, il montre alors que ce n'est pas le cas (p. 283).

La question du déterminisme est au cœur même du chapitre, "Theories: Mediatization and Media Ecology", où Lynn Schfield Clark explore la relation entre diverses théories des médias et 
l'approche de l'écologie des médias dont Marshall McLuhan, Neil Postman, Walter Ong et Harold Innis sont les principaux représentants. Notons qu'au cœur de cette approche, il y a la théorie du médium. Le médium, envisagé ici comme prolongement de nos facultés physiques et mentales, influencerait de manière fondamentale nos perceptions, nos sentiments, nos valeurs, notre rapport à la réalité. En clair, l'écologie des médias va être marquée par une posture déterministe mettant de l'avant le pouvoir transformationnel des médias. Cependant, dans sa synthèse des travaux en écologie des médias, Lynn Schofield Clark trouve à l'approche quelques affinités avec le courant socioconstructiviste et plus largement avec la médiatisation. En l'occurrence, elle soutient que

McLuhan's theories of technologies as extension of human capabilities suggest common ground with cybernetics, and particularly cyborg and the posthuman. As such, media ecology can point to fruitful avenues for further exploration that avoids linear and causal assumptions in favour of an approach that assumes change is recursive, continuous, and co-created as part of a cybernetic circuit (p. 98).

Ce n'est pas d'aujourd'hui qu'on tente de nuancer l'adhésion de l'écologie des médias à une "épistémologie déterministe ${ }^{1}$ ". Gertrude J. Robinson se prêtait déjà à l'exercice quand elle affirmait que, chez Innis, «le changement social s'élabore selon un processus infiniment plus complexe, dans lequel les technologies de communication jouent un rôle crucial ${ }^{2}$ ".

\section{Quelques remarques}

Comment saisir le contexte actuel de saturation médiatique ainsi que les transformations qu'elle semble provoquer? C'est sans doute là la question centrale de cet ouvrage. Mais une question qui en appelle une autre, celle de la définition même du concept de média, malheureusement insuffisamment débattue dans le volume. Aussi, Knut Lundby pose comme jalons clé de la

1 Proulx, Serge, "Écologie des médias : une ouverture critique ", dans Patrick Yves Badillo (dir.), Écologie des médias, Bruxelles, Bruylant, 2008, p. 71-79.

2 Paquet-Sévigny, Thérèse, Communication et développement international, Montréal, Presses de l’Université du Québec, 1996, p. 48. 
recherche en médiatisation un certain nombre de travaux : ceux de Harrold Innis au début des années 1950, ceux de Marshall McLuhan, avec notamment sa "théorie des médiums ", de Jesús Martín-Barbero sur la "médiation » dans les mouvements populaires en Amérique latine, les travaux de John $\mathrm{B}$. Thompson à qui on doit le concept de "mediazation of culture ", de même que ceux de Roger Sylverstone qui s'est intéressé à l'impact du processus même de communication sur l'environnement culturel (p. 2, 3). Une trajectoire qui me semble par trop sélective, puisqu'elle occulte les travaux fondateurs en étude des médias. En l'occurrence, ceux de l'École de Frankfort (Theodor Adorno, Max Horkheimer, Herbert Marcuse) sur la propagande et l'industrie culturelle, dont les chercheurs, animés par une intuition béhavioriste, vont postuler un effet direct des médias sur le public. Elle passe aussi sous-silence l'École de Columbia (Paul F. Lazarsfeld, Elihu Katz) dont les travaux empiriques remettront en cause les postulats béhavioristes et ouvriront sur le paradigme de l'effet limité des médias sur les comportements des individus. Par ailleurs, le volume regroupe diverses analyses et perspectives de recherche en médias et société sous le vocable médiatisation. Cependant, la question de savoir comment il faut envisager ce concept reste irrésolue. La médiatisation est-elle un phénomène? Une théorie? Une approche? Peut-être est-ce simplement une hypothèse, ou même une croyance. Quoi qu'il en soit, la proposition d'Eric Rothenbuhler mérite considération :

If we take mediatization as a topic, a research question, or a perspective rather than a specific theory, then we can benefit from this wide range of conceptual associations among the works of these and other scholars and avoid the need to debate the details. In this perspective, mediatization, or whatever you prefer to call it, is a term for drawing attention to how social activities begin to change in the presence of the media; it is a useful way to draw attention to a certain class of media consequences; it is a topic to investigate rather than a prediction or a knowledge claim (p. 280). 


\section{Bibliographie}

Paquet-Sévigny, Thérèse, Communication et développement international, Montréal, Presses de l'Université du Québec, 1996.

Proulx, Serge, "Écologie des médias : une ouverture critique », dans Patrick Yves Badillo (dir.), Écologie des médias, Bruxelles, Bruylant, 2008, p. $71-79$. 